\title{
Relevant Scale of Structural Disorder for Carrier Localization in $\mathrm{YBa}_{2} \mathrm{Cu}_{3} \mathrm{O}_{6.9}{ }^{1}$
}

\author{
Andrea Gauzzi ${ }^{2,3}$ and Davor Pavuna ${ }^{2}$
}

Received 9 December 1996

Resistivity measurements on a large number of $\mathrm{YBa}_{2} \mathrm{Cu}_{3} \mathrm{O}_{6.9}$ films with reduced long-range order of the crystal show that both normal and superconducting carriers are localized within the coherence length $r_{c}$ of the crystal, if $r_{c}$ is reduced below $\approx 10 \mathrm{~nm}$. This result suggests that the localized character of the metallic state observed experimentally in cuprates arises from local structural inhomogeneities and disorder in the nm scale, independent of the nature of the metallic and superconducting states in the $\mathrm{CuO}_{2}$ plane.

KEY WORDS: Copper oxide superconductors; transport properties; disorder; localization.

Unlike in the case of ordinary metals with conduction bands formed by $s$ - or $p$-orbitals, the metallic state of cuprates is characterized by the pronounced directionality of the $3 d_{x^{2}}-y^{2}$ orbitals of copper forming the conduction band in the $\mathrm{CuO}_{2}$ planes. The relevance of these orbitals in determining the electronic structure of these materials is put into evidence by the validity of a simple tight-binding approximation to account for the main features of the characteristic square-shaped Fermi surface of the $\mathrm{CuO}_{2}$ planes [1]. Such pronounced directionality implies that the longrange order of the crystal is necessary to obtain charge delocalization, especially because disorder-induced localization is favored by the quasi-two-dimensionality of the electronic states in the above planes. This circumstance accounts for the tendency toward charge localization observed in several compounds; experimental evidence of this tendency is given, for example, by the characteristic upturn of the temperature-dependent resistivity in the vicinity of the superconducting transition observed in the underdoped regime and already reported in the original work by Bednorz and Müller in the $\mathrm{La}-\mathrm{Ba}-\mathrm{Cu}-\mathrm{O}$ system [2]. This feature

\footnotetext{
${ }^{1}$ To the memory of Nevil Mott (1905-1996).

${ }^{2}$ Department of Physics, Swiss Federal Institute of Technology at Lausanne (EPFL), CH-1015 Lausanne, Switzerland.

${ }^{3}$ Present address: MASPEC-CNR, Via Chiavari 18/A, I-43100 Parma, Italy.
}

of the resistivity has been more recently observed also at low temperatures in underdoped $\mathrm{La}_{2-x} \mathrm{Sr}_{x} \mathrm{CuO}_{4}$ [3] and in $\mathrm{Bi}_{2} \mathrm{Sr}_{2} \mathrm{CuO}_{y}$ [4] by suppressing the superconducting transition in a strong magnetic field. Further evidence for charge localization in a superconducting cuprate has been reported in $\mathrm{Pr}$-substituted $\mathrm{BiSrCa}$ $\mathrm{CuO}$ by Hall effect measurements [5]. This kind of experimental observations has led to proposals of nonconventional or non-Fermi liquid mechanisms of charge transport and of superconductivity, such as the polaron model developed by Alexandrov and Mott [6] or the RVB theory by Anderson [7]. To establish the validity and the theoretical implications of such generalized approaches, it is important to verify to which extent the aforementioned localized character of the charges in cuprates is determined by local structural and electronic inhomogeneities and by disorder, independent of the nature of the metallic state of the $\mathrm{CuO}_{2}$ planes. This is because it has been established experimentally by various experimental methods that the above inhomogeneities and shortrange order of the crystal are unavoidable in cuprates even in best-quality samples because of nonstoichiometry, latent structural instabilities, and the tendency toward structural and electronic phase separation [8]. In order to elucidate the above issue, here we shall discuss the role of the coherence length $r_{c}$ of the crystal in determining both transport and superconducting properties. For this purpose, we 
shall analyze experimental data of resistivity and of superconducting transition on a large number of $\mathrm{YBa}_{2} \mathrm{Cu}_{3} \mathrm{O}_{6.9}$ films with reduced long-range order of the crystal. The fundamental result of our analysis indicates that $r_{c}$, which is determined experimentally from the width of $\mathrm{X}$-ray diffraction rocking curves, cuts off the localization length of the mobile charges in the normal state and the divergence of the superconducting coherence length $\xi$ at the superconducting transition. We therefore conclude that in $\mathrm{YBa}_{2} \mathrm{Cu}_{3} \mathrm{O}_{6.9}, r_{c}$ is a fundamental characteristic length which determines the crossover from metallic to localized regime of normal transport and the width of the transition region.

The experimental data analyzed in this work has been reported in detail elsewhere $[9,10]$. In summary, we have prepared a series of fully doped $(x \approx 0.9) \approx 40 \mathrm{~nm}$ thick, $c$-axis-oriented $\mathrm{YBa}_{2} \mathrm{Cu}_{3} \mathrm{O}_{6+x}$ films epitaxially grown on (100) $\mathrm{SrTiO}_{3}$ and $\mathrm{LaAlO}_{3}$ by ion-beam sputtering. The films have been grown with different degrees of lattice order by slightly varying the growth temperature, while all the other deposition parameters were held constant. X-ray diffraction data indicate that the long-range order of the lattice is progressively reduced as the growth temperature deviates from an optimum value of $\approx 675^{\circ} \mathrm{C}$, while the oxygen content $x$ remains constant and equal to $\approx 0.9$. Under this oxygen doping regime, the variations of the superconducting critical temperature $T_{c}$ associated with local variations of oxygen ordering in the basal planes which are possible in underdoped $(x<0.9)$ samples $[11,12]$ are prevented. This is because the number of possible schemes of oxygen ordering in the basal planes is small for $x \approx 0.9$ and all these schemes are similar to the ordering scheme of the ortho-II structure. Thus, the spatial distribution of oxygen atoms within these planes is expected to be homogeneous in the local (nm) scale, independent of the amount of disorder. This hypothesis is confirmed by the fact that the onset of the superconducting critical temperature is constant and equal to the maximum value of $\approx 92 \mathrm{~K}$ in all films.

The reduction of the long-range order of the lattice can be conveniently expressed in a quantitative manner in terms of the coherence length $r_{c}$ of the crystal. This length can be determined from the width $\Delta \vartheta$ of X-ray diffraction rocking curves. In the case of Lorentzian broadening, which corresponds to our case, a straightforward calculation yields [9]

$$
r_{c}=\frac{1}{\pi} \frac{d}{l \Delta \vartheta}
$$

where $\Delta \vartheta$ is the full width at half maximum, $d$ is the distance between adjacent diffracting planes of the Bragg reflection, and $l$ is the order of this reflection. Our present data refer to the (005) reflection, hence $d=1.17 \mathrm{~nm}$ is the $c$-axis parameter of $\mathrm{YBa}_{2} \mathrm{Cu}_{3} \mathrm{O}_{6.9}$, $l=5$, and Eq. (1) expresses $r_{c}$ in the $a b$-plane.

By using Eq. (1), we have found that the effects of growth-induced reduction of $r_{c}$ on the resistivity and on the superconducting transition appear for values of $r_{c}$ smaller than $\approx 10 \mathrm{~nm}$; these effects manifest themselves as an increase of the resistivity, the appearance of a saturation of the resistivity at high temperatures, and a broadening of the superconducting transition $[9,10]$. The first two effects are interpreted as the signature of weak localization of the mobile charges. A quantitative analysis is difficult in the absence of an appropriate model of charge transport in the normal state for cuprates. Nevertheless, further insight into the phenomenon is provided by noting that the mean-free path at room temperature in best-quality films with $T_{c} \approx 92 \mathrm{~K}$ is estimated to be $\approx 12 \mathrm{~nm}$ within the framework of a simple Drude model, by taking a room-temperature resistivity of $\approx 250 \mu \Omega \mathrm{cm}$ and a carrier density of $\approx 5 \times 10^{21}$ hole/ $\mathrm{cm}^{3}$. This simple estimation suggests that localization effects appear as soon as the coherence length $r_{c}$ of the crystal becomes smaller than the mean-free path in the absence of disorder. In other words, the normal carriers are localized within a characteristic length of the order of $r_{c}$. A quantitative analysis is possible for the data of broadening $\Delta T_{c}$ of the transition caused by the disorder. We apply the Ginzburg-Landau theory, since mean-field theories are expected to apply to homogeneously disordered media. The applicability of this theory to our $\Delta T_{c}$ data is supported by the dependence of this data on the reduction of $r_{c}$ following the empirical scaling relation

$$
\Delta T_{c} \propto r_{c}^{-2}
$$

which is explained by a cut-off effect of $r_{c}$ on the divergence of the temperature-dependent GinzburgLandau coherence length $\xi \equiv \xi_{0}\left|1-T / T_{c}\right|^{-1 / 2}$ of the order parameter. Such cut-off effect follows from the aforementioned effect of localization of the normal carriers within a characteristic length $r_{c}$. This effect applies also to the superconducting carriers, since the superconducting wave function is built from single electron states. Hence, we argue that for cuprates the following inequality holds:

$$
\xi(T) \lesssim r_{c}
$$

where we have emphasized that $\xi$ depends on temperature. We summarize the physical meaning of Eqs. 
Fig. 1. Diagram representing the cutoff effect of the crystal coherence length $r_{r}$ on the divergence of the Ginzburg-Landau superconducting coherence length $\xi$ at $T_{r}$. The solid line represents this divergence and also the broadening $\Delta T_{c}$ of the superconducting transition for different values of the ratio $r_{c} / \xi_{0}$, according to Eq. (2). $T_{c, x}$ indicates the mean-field critical temperature. Different limiting cases $r_{c} \gg \xi_{0}, r_{c} \sim \xi_{0}$, and $r_{c} \ll \xi_{0}$ are represented schematically. The shaded area represents the case of conventional superconductors with large values of $\xi_{0}$, which implies $r_{1} \ll \xi_{0}$ in both limits of amorphous and good crystalline samples. This explains the small effect of disorder-induced reduction of $T_{c}$ observed in these superconductors (see [13]). On the other hand. cuprates correspond to the other two limiting cases $r_{c} \gg \xi_{0}$ in good crystalline samples and $r . \sim \check{\zeta}_{0}$ in samples with reduced long range order. The comparison of this diagram with the data on $\mathrm{YBa}_{2} \mathrm{Cu}_{3} \mathrm{O}_{6.9}$ films cited in $[10]$ and in the text shows that such data cover the transition region between these two regimes.

(2) and (3) in the diagram of Fig. 1, where we represent the broadening of $T_{c}$ corresponding to a given ratio of the dimensionless variable $r_{c} / \xi_{0}$. In particular, this diagram accounts for the fact that in conventional superconductors with low $T_{c}$ (and therefore large $\xi_{0}$ ) the effects of lattice disorder on $T_{c}$ are usually small [13], since in these superconductors the inequality $\xi_{0} \gg r_{c}$ holds for both good crystalline and disordered (or even amorphous) samples. On the other hand, the large effects discussed here on $\mathrm{YBa}_{2} \mathrm{Cu}_{3} \mathrm{O}_{6.9}$ are explained by the validity of the opposite inequality $\xi_{0} \leqslant r_{c}$ which holds in general for all cuprates.

In conclusion, our analysis of resistivity and $T_{c}$ measurements on a large number of $\mathrm{YBa}_{2} \mathrm{Cu}_{3} \mathrm{O}_{6.9}$ films with reduced long-range order shows that $r_{c}$ is a fundamental length that governs the crossover from metallic to localized regime of normal transport and the width of the transition region in this compound. Quantitative account for our data is given by the cutoff effect of $r_{c}$ on the divergence of $\xi$ at the transition, which leads to expressing the empirical scaling relation (2) as a function of the dimensionless variable $\xi_{0} / r_{c}$ only. This result suggests that the validity of this scaling might be extended to other cuprates and to other systems where the second-order phase transition is governed by an order parameter with short $\xi_{0}$.
$A$ Perfect lattice

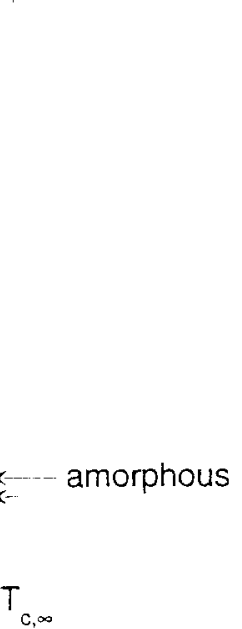

We acknowledge I. Bozovič, I. Wooldridge, and M. A. Howson for useful discussions and J. Berrocosa for his technical help. We gratefully acknowledge financial support by the Swiss National Research Foundation.

\section{REFERENCES}

1. L. F. Mattheiss, Phys. Rev. Lett. 58, 1028 (1987); J. Labbé and J. Bok, Europhys. Lett. 3, 1237 (1987).

2. J. G. Bednorz and K. A. Müller, Z. Phys. B 64, 189 (1986)

3. G. S. Boebinger, Y. Ando, A. Passner, T. Kimura, M. Okuya, J. Shimoyama, K. Kishio, K. Tamasaku, N. Ichikawa, and S. Uchida, Phvs. Rev. Lett. 77, 5417 (1996).

4. Y. Ando. G. S. Boebinger, A. Passner, N. L. Wang, C. Geibel, and F. Steglich, Phys. Rev. Lett. 77, 2065 (1996).

5. B. Beschoten, S. Sadewasser, G. Güntherodt, and C. Quitmann, Phys. Rev. Lett. 77, 1837 (1997)

6. A. S. Alexandrov and N. Mott, Rep. Prog. Phys. 57, 1197 (1994).

7. P. W. Anderson, Science 256, 1526 (1992).

8. For a review, see Phase Separation in Cuprate Superconductors, K. A. Müller and G. Benedek, eds. (World Scientific, Singapore, 1993), and references therein.

9. A. Gauzzi and D. Pavuna, Appl. Phys. Lett. 66, 1836 (1995)

10. A. Gauzzi, B. J. Jönsson, A. Clerc-Dubois, and D. Pavuna, preprint (1997)

11. J. D. Jorgensen, S. Pei, P. Lightfoot, H. Shi, A. P. Paulikas, and B. W. Veal, Physica C 58, 571 (1990).

12. H. Shaked. J. D. Jorgensen, B. A. Hunter, R. L. Hitterman, A. P. Paulikas, and B. W. Veal, Phys. Rev. B 51, 547 (1995).

13. See, for example, K. Knorr and N. Barth, Solid State Commun 6, 791 (1968); G. Bergmann, Z. Phys. 228, 25 (1969); G. Ziemba and G. Bergmann, ibid, 237, 410 (1970). 